\title{
Comment on "A Prospective Study on Electrocardiographic Findings of Patients with Organophosphorus Poisoning”
}

\author{
Farzad Gheshlaghi
}

Published online: 25 April 2012

(C) Springer Science+Business Media, LLC 2012

\section{Dear Editor,}

I read with interest the study conducted by Vijayakumar and colleagues entitled " A Prospective Study on Electrocardiographic Findings of Patients with Organophosphorus Poisoning " published in your journal [1]. The authors concluded that acute organophosphorus poisoning is associated with ventricular arrhythmias, tachycardia and bradycardia and attributes mild myocardial ischemia.

I think the following suggestions likely to be worthwhile for future similar studies:

First: In methodology section of the article, any history of resent use of cardioactive drugs specifically those which may be prolong QT interval like methadone should be consider as exclusion criteria and clearly written in that section.

Second: Considering the average patients, admission time was $1 \mathrm{~h}$ and $10 \mathrm{~min}$; therefore, severity of poisoning is likely to be different in patients, so it is better to matching these cases according to poisoning severity score.

Third: To illustrate the significance of electrocardiographic findings of patients with organophosphorus poisoning, the authors explain the correlation between these changes and patients outcome.

Thanks for this interesting paper.

Conflicts of interest None.

\section{Reference}

1. Vijayakumar, S., Fareedullah, M., Ashok Kumar, E., \& Mohan Rao, K. (2011). A prospective study on electrocardiographic findings of patients with organophosphorus poisoning. Cardiovascular Toxicology, 11, 113-117.
F. Gheshlaghi

Department of Clinical Toxicology and Forensic Medicine,

School of Medicine, Isfahan University of Medical Sciences,

Esfahan, Iran

F. Gheshlaghi $(\bowtie)$

Isfahan Clinical Toxicology Research Center, Isfahan Univetsity

of Medical Sciences, Esfahan, Iran

e-mail: gheshlaghi@med.mui.ac.ir 